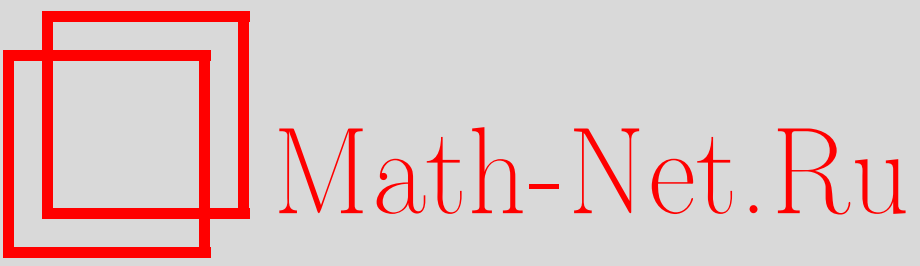

А. Я. Мальцев, Усреднение слабо-нелокальных симплектических структур, УМН, 2004, том 59, выпуск 2, 193-194

DOI: https://doi.org/10.4213/rm728

Использование Общероссийского математического портала Math-Net.Ru подразумевает, что вы прочитали и согласны с пользовательским соглашением

http://www.mathnet.ru/rus/agreement

Параметры загрузки:

IP: 44.207 .124 .84

26 апреля 2023 г., 10:30:48 


\title{
УСРЕДНЕНИЕ СЛАБО-НЕЛОКАЛЬНЫХ СИМПЛЕКТИЧЕСКИХ СТРУКТУР
}

\author{
А. Я. МАЛЬЦЕВ
}

Мы рассматриваем общие слабо-нелокальные симплектические структуры [1], имеющие вид

$$
\Omega_{i j}(x, y)=\sum_{k \geqslant 0} \omega_{i j}^{(k)}\left(\varphi, \varphi_{x}, \ldots\right) \delta^{(k)}(x-y)+\sum_{s=1}^{g} e_{s} \frac{\delta H^{(s)}}{\delta \varphi^{i}(x)} \nu(x-y) \frac{\delta H^{(s)}}{\delta \varphi^{j}(y)},
$$

где $i, j=1, \ldots, n, \nu(x-y)=(1 / 2) \operatorname{sign}(x-y), \delta^{(k)}(x-y)=\partial^{k} / \partial x^{k} \delta(x-y), e_{s}= \pm 1, H^{(s)}[\varphi]=$ $\int_{-\infty}^{+\infty} h^{(s)}\left(\varphi, \varphi_{x}, \ldots\right) d x$ и $\Omega_{i j}(x, y)$ задает замкнутую 2-форму на пространстве функций $\varphi(x)=$ $\left(\varphi^{1}(x), \ldots, \varphi^{n}(x)\right)$. Мы будем рассматривать здесь локальные эволюционные системы

$$
\varphi_{t}^{i}=Q^{i}\left(\varphi, \varphi_{x}, \ldots\right)
$$

допускающие симплектическую структуру (1) с гамилттонианом $H=\int_{-\infty}^{+\infty} h\left(\varphi, \varphi_{x}, \ldots\right) d x$. (Функционалы $H^{(s)}[\varphi]$ должны в этом случае являться законами сохранения для системы (2), так что $h_{t}^{(s)} \equiv \partial_{x} J^{(s)}$ для некоторых $J^{(s)}\left(\varphi, \varphi_{x}, \ldots\right)$.)

Мы будем рассматривать хорошо известньй метод усреднения Уизема [2]-[5] для системы (2), дающий нелинейную систему уравнений на медленно промодулированные параметры $U^{\nu}$ $m$-фазных решений $(2): \varphi^{i}(x, t)=\Phi^{i}\left(\omega(U) t+k(U) x+\theta_{0}, U^{1}, \ldots, U^{N}\right)$, имеющую вид

$$
U_{T}^{\nu}=V_{\mu}^{\nu}(U) U_{X}^{\mu}, \quad \nu, \mu=1, \ldots, N
$$

(система Уизема). Функции $\Phi^{i}(\theta, U)$ являются здесь $2 \pi$-периодическими функциями по отношению к переменным $\left(\theta^{1}, \ldots, \theta^{m}\right)=\theta$ и зависят от дополнительных параметров $U^{1}, \ldots, U^{N}$. Векторы ( $m$-компонентные) $\omega(U)$ и $k(U)$ играют роль частот и волновых чисел для квазипериодических решений. В методе Уизема параметры $U^{\nu}$ становятся функциями “медленных переменных" $X=\varepsilon x, T=\varepsilon t, \varepsilon \rightarrow 0$ и система (3) дает необходимые условия для построения соответствуюшего асимптотического решения (2).

Мы построим здесь процедуру “усреднения" симплектической структуры (1), дающую слабо-нелокальную симплектическую структуру гидродинамического типа для системы (3).

ОПРедЕЛЕниЕ 1. Мы называем слабо-нелокальной симплектической структурой гидродинамического типа слабо-нелокальную симплектическую структуру, имеющую вид

$$
\Omega_{\nu \mu}(X, Y)=\sum_{s, k=1}^{M} \kappa_{s k} \frac{\partial f^{(s)}}{\partial U^{\nu}}(X) \nu(X-Y) \frac{\partial f^{(k)}}{\partial U^{\mu}}(Y)
$$

где $f^{(s)}(U)$ - некоторые функции переменных $U^{1}, \ldots, U^{N}$ и $\kappa_{s k}$-некоторая постоянная симметричная билинейная форма $(\nu, \mu=1, \ldots, N)$.

Определим "усреднение" любой локальной функции $f\left(\varphi, \varphi_{x}, \ldots\right)$ согласно формуле $\langle f\rangle(U)=\int_{0}^{2 \pi} \cdots \int_{0}^{2 \pi} f\left(\Phi(\theta, U), k^{\alpha}(U) \Phi_{\theta^{\alpha}}(\theta, U), \ldots\right)\left(d^{m} \theta /(2 \pi)^{m}\right)$. Определим также функции $T_{\alpha}^{(s)}\left(\varphi, \varphi_{x}, \varphi_{\theta^{\alpha}}, \ldots\right)=\sum_{k \geqslant 1} \sum_{p=0}^{k-1}(-1)^{p}\left(\partial h^{(s)} / \partial \varphi_{k x}^{i}\right)_{p x} \varphi_{\theta^{\alpha},(k-p-1) x}$.

Лемма 1. 1) Для любой симплектической структуры (1) имеют место следующие соотношения:

$$
\begin{gathered}
\varphi_{\theta^{\alpha}}^{i} \sum_{k \geqslant 0} \omega_{i j}^{(k)}\left(\varphi, \varphi_{x}, \ldots\right) \varphi_{\theta^{\beta}, k x}^{j}+\sum_{s=1}^{g} e_{s}\left(h_{\theta^{\beta}}^{(s)} T_{\alpha}^{(s)}-h_{\theta^{\alpha}}^{(s)} T_{\beta}^{(s)}+\left(T_{\alpha}^{(s)}\right)_{x} T_{\beta}^{(s)}\right) \\
\equiv \frac{\partial}{\partial \theta^{\gamma}} Q_{\alpha \beta}^{\gamma}(\varphi, \ldots)+\frac{\partial}{\partial x} A_{\alpha \beta}(\varphi, \ldots)
\end{gathered}
$$

для некоторых функций $Q_{\alpha \beta}^{\gamma}(\varphi, \ldots), A_{\alpha \beta}(\varphi, \ldots)$.

Работа выполнена при поддержке Гранта Президента РФ (MK-1375.2003.02) и Российского фонда фундаменталшных исследований (грант № 03-01-00368). 
2) Функции $A_{\alpha \beta}(\varphi, \ldots)$ (определеннье с точностью до констант) могут быть определень таким образом, что $A_{\alpha \beta}(\varphi, \ldots) \equiv 0$ для любой $\varphi(\theta, x)$, зависящей только от $x$ ( и постоянной по $\theta$ при каждом фиксированном $x$ ).

Мы предположим теперь, что имеются $m$ линейно независимых коммутирующих потоков $\varphi_{t^{\alpha}}^{i}=Q_{\alpha}^{i}\left(\varphi, \varphi_{x}, \ldots\right), \alpha=1, \ldots, m$, системы $(2)$, оставляющих инвариантным семейство квази-периодических решений и генерирующих на нем линейные сдвиги начальных фаз $\theta_{0}^{\alpha}$ с частотами $\omega_{(\alpha)}(U)$. Мы требуем также, чтобы функционалы $H^{(s)}[\varphi]$ являлись законами сохранения для потоков $\varphi_{t^{\alpha}}^{i}$, так что $h_{t^{\alpha}}^{(s)} \equiv \partial_{x} J_{\alpha}^{(s)}$ для некоторых локальных функций $J_{\alpha}^{(s)}\left(\varphi, \varphi_{x}, \ldots\right)$. Кроме того, мы требуем, чтобы матрица $\left\|\omega_{(\alpha)}^{\beta}(U)\right\|$ была невырожденной и имела обратную $\left\|\gamma_{\alpha}^{\beta}(U)\right\|$, так что $\gamma_{\delta}^{\beta}(U) \omega_{(\alpha)}^{\delta}(U) \equiv \delta_{\alpha}^{\beta}$.

ТеОРема 1. При выполнении всех сформулированных выше условий система Уизема (3) имеет слабо-нелокальную симплектическую структуру гидродинамического ти$n a$

$$
\begin{aligned}
\Omega_{\nu \mu}(X, Y)=\sum_{\alpha=1}^{m} & \left(\frac{\partial k^{\alpha}}{\partial U^{\nu}}(X) \nu(X-Y) \frac{\partial I_{\alpha}}{\partial U^{\mu}}(Y)+\frac{\partial I_{\alpha}}{\partial U^{\nu}}(X) \nu(X-Y) \frac{\partial k^{\alpha}}{\partial U^{\mu}}(Y)\right) \\
& +\sum_{s=1}^{g} e_{s} \frac{\partial\left\langle h^{(s)}\right\rangle}{\partial U^{\nu}}(X) \nu(X-Y) \frac{\partial\left\langle h^{(s)}\right\rangle}{\partial U^{\mu}}(Y)
\end{aligned}
$$

с гамильтонианом $H=\int_{-\infty}^{+\infty}\langle h\rangle d X$, где "переменнье действия" $I_{\alpha}(U)$ определень посредством формул:

$$
\begin{aligned}
\frac{\partial I_{\alpha}}{\partial U^{\nu}} & =\frac{\partial k^{\beta}}{\partial U^{\nu}}\left[-\left\langle A_{\alpha \beta}\right\rangle+\gamma_{\alpha}^{\delta} \sum_{s=1}^{g} e_{s}\left(\left\langle T_{\beta}^{(s)} J_{\delta}^{(s)}\right\rangle-\left\langle T_{\beta}^{(s)}\right\rangle\left\langle J_{\delta}^{(s)}\right\rangle\right)\right. \\
& \left.-\frac{1}{2} \gamma_{\alpha}^{\delta} \gamma_{\beta}^{\zeta} \sum_{s=1}^{g} e_{s}\left(\left\langle J_{\delta}^{(s)} J_{\zeta}^{(s)}\right\rangle-\left\langle J_{\delta}^{(s)}\right\rangle\left\langle J_{\zeta}^{(s)}\right\rangle\right)\right]+\left\langle\Phi_{U^{\nu}}^{i} \sum_{k \geqslant 0} \omega_{i j}^{(k)}(\varphi, \ldots) \varphi_{\theta^{\alpha}, k x}^{j}\right\rangle \\
& -\sum_{s=1}^{g} e_{s}\left\langle\Phi_{U^{\nu}}^{i} \frac{\delta H^{(s)}}{\delta \varphi^{i}(x)} T_{\alpha}^{(s)}\right\rangle+\sum_{s=1}^{g} e_{s} \gamma_{\alpha}^{\beta}\left(\left\langle\Phi_{U^{\nu}}^{i} \frac{\delta H^{(s)}}{\delta \varphi^{i}(x)} J_{\beta}^{(s)}\right\rangle-\left\langle\Phi_{U^{\nu}}^{i} \frac{\delta H^{(s)}}{\delta \varphi^{i}(x)}\right\rangle\left\langle J_{\beta}^{(s)}\right\rangle\right)
\end{aligned}
$$

и функиии $A_{\alpha \beta}$ нормированы в соответствии с леммой 1.

Добавим также, что функционалы $\int_{-\infty}^{+\infty} I_{\alpha} d X$, так же как и $\int_{-\infty}^{+\infty} k^{\alpha} d X, \int_{-\infty}^{+\infty}\left\langle h^{(s)}\right\rangle d X$ и $H=\int_{-\infty}^{+\infty}\langle h\rangle d X$ являются законами сохранения для системы Уизема (3).

\section{СПИСОК ЛИТЕРАТУРЫ}

[1] A. Ya. Maltsev, S. P. Novikov // Phys. D. 2001. V. 156. №1-2. P. 53-80. [2] G. B. Whitham. Linear and Nonlinear Waves. New York: Wiley, 1974. [3] J.C. Luke // Proc. Roy. Soc. London Ser. А. 1966. V. 292. №1430. P. 403-412. [4] Б. А. Дубровин, С. П. Новиков // УМН. 1989. Т. 44. №6. С. 29-98. [5] B. A. Dubrovin, S. P. Novikov // Soviet Sci. Rev. Sect. C Math. Phys. 1993. V. 9. Part 4. P. 1-136. 